\title{
SEARCH: An SDN-enabled Approach for Vehicle Path-Planning
}

\author{
Omar Sami Oubbati, Member, IEEE, Mohammed Atiquzzaman, Senior Member, IEEE, \\ Pascal Lorenz, Senior Member, IEEE, Abdullah Baz, Senior Member, IEEE, \\ and Hosam Alhakami, Senior Member, IEEE
}

\begin{abstract}
With increasing vehicle density and the growth of accidents in urban areas, navigation management becomes a serious problem. Even though there is a multitude of navigation systems, ambulances, taxis, or even ordinary vehicles, sometimes find it challenging to reach their destinations on time. There are two main reasons for this difficulty: (i) lack of local knowledge of the area of navigation solutions and (ii) their inflexibility against unforeseeable situations that may occur on the roads. Indeed, the majority of navigation solutions are based only on the distance, the journey time, or even statistics related to the density of vehicles to plan the full paths, while neglecting the dynamic nature of the vehicle traffic. Also, their respective centralized architectures are unable to monitor both the traffic and unexpected events continuously and in real-time without not being overloaded by the flow of message exchanges between the road entities and the central processing entity. To address these issues, we propose in this paper a novel three-tier architecture, called SDN-enabled Approach for Vehicle Path-Planning (SEARCH), to enhance the situation awareness on urban roads, efficiently collect traffic information in real-time, and decide the best navigation strategy. The proposed architecture exploits Unmanned Aerial Vehicles (UAVs), Vehicular Ad hoc Networks (VANETs), 5G based cellular systems, and Software-Defined Networking (SDN) to provide better and faster communication to changing road conditions. Based on these technologies, some parameters related to vehicles and driving environments, such as speed, distance, traffic jams, incidents, and travel flow, are efficiently collected and dynamically exploited to achieve faster paths between any existing pairs of locations. Furthermore, the deployed architecture of SEARCH can provide sufficient bandwidth to support all data traffic needs to update vehicles during their journey efficiently and in real-time. To evaluate the performance of our architecture, we conduct a series of simulations and perform a set of comparisons with relevant route planning algorithms. We found that the proposed architecture works effectively in terms of saving on driving time to reach any target destinations.
\end{abstract}

Index Terms-5G; SDN; Traffic management; VANET; Path planning.

Copyright (c) 2020 IEEE. Personal use of this material is permitted. However, permission to use this material for any other purposes must be obtained from the IEEE by sending a request to pubs-permissions@ieee.org.

O.S. Oubbati is with the Computer Science and mathematics Laboratory, University of Laghouat, Algeria. E-mail: s.oubbati@lagh-univ.dz.

M. Atiquzzaman is with the University of Oklahoma, Norman, OK USA. E-mail: atiq@ou.edu

P. Lorenz is with the University of Haute Alsace, IUT Colmar, France. E-mail: pascal.lorenz@uha.fr

A. Baz is with the Department of Computer Engineering, College of Computer and Information Systems, Umm Al-Qura University, Makkah, Saudi Arabia. Email: aobaz01@uqu.edu.sa

H. Alhakami is with the Department of Computer Science, College of Computer and Information Systems, Umm Al-Qura University, Makkah, Saudi Arabia. Email: hhhakam@uqu.edu.sa

\section{INTRODUCTION}

The last decade has witnessed a severe increase in congestion on our roads. Indeed, different statistics across the web show that traffic congestion can negatively affect the economic growth of cities by losing travel time, wasted money due to loss of productivity, and waste of fuel [1]. Even worse, the number of vehicles continues to increase at a rate of four million vehicles a year, especially in metropolitan areas where the majority of the world's population lives [2]. As a result, a considerable effort has been undertaken by researchers to add new infrastructures (e.g., dedicated lanes for buses) to minimize the congestion [3]. Nevertheless, the impact of this classical solution is restricted because the speed of construction of new roads is much slower than the increase in the number of new vehicles. To adapt to this situation and traveling without wasting time on roads, scientists try to design path planning mechanisms to allow road-users to avoid traffic congestion whenever possible. However, most current path planning solutions are based exclusively on the shortest distance or shortest time path algorithms, without considering the impact of future traffic conditions during the duration of the trip. Consequently, scientists have looked heavily into the possibility of using dynamic path planning based on real-time traffic information, which gives rise to the following challenges:

- How to efficiently collect traffic information from the roads in real-time?

- How to detect any incidents or temporary events (e.g., road repair) that can occur at any time on the roads?

- When is the right time to make path re-planning decisions?

- How to accurately estimate the journey time of a given vehicle?

- What is the best network architecture that provides optimal coverage, saves bandwidth, reduces latency, and maximizes throughput?

To overcome all these challenges, a set of emerging technologies, such as 5G based cellular systems, Unmanned Aerial Vehicles (UAVs), Vehicular Ad hoc Networks (VANETs), and Software-Defined Networking (SDN), are expected to answer all those questions and significantly mitigate urban traffic congestion. Indeed, 5G technology offers enhanced scalability and higher bandwidths to support different applications dealing with the traffic congestion issues in the roads [4], [5]. VANETs have been heavily involved 
in nearly all safety and comfort applications, such as crash avoidance, autonomous driving, cooperative driving, collective situational awareness, and even natural language processing [6]. Therefore, VANETs are already approaching deployment, which would make the roads safer, the travel experience more pleasant, and the transportation system more efficient [7], [8]. To assist 5G cellular networks and VANETs, UAVs are well equipped with the required devices to ensure coverage, collect traffic information, and explore different geographical areas, for example, through aerial photography [9]. To top it all, SDN technology can effectively manage all these networks by bringing flexibility, scalability, and programmability, and thus exploit the available network resources more efficiently [10], [11]. The combination of these technologies can satisfy the requirements of various applications, such as vehicle path-planning schemes that have and continue to receive significant attention from the research community [12]. These schemes play a crucial role in reducing traffic congestion, thereby enhancing the overall economy in terms of energy consumption, time, and productivity. Indeed, they consist of computing the shortest-time path between a pair of locations within a road network while considering several dynamic parameters, such as the real-time traffic congestion level, unexpected incidents, and road layout. Therefore, designing a path planning algorithm is regarded as a challenging task because it should consider the specific issues that characterize any urban areas.

Motivated by the challenges mentioned above and considering the advantages of employing such technologies in urban traffic management, we propose an SDN-based architecture for vehicle path planning in urban areas. The emerging SDN technology significantly enhances the propagation of messages in VANETs, where a remote server and vehicles play the roles of a central controller (i.e., SDN controller) and switches, respectively. The proposed architecture consists of a path planning algorithm to allow all kinds of vehicles to dynamically estimate shorter time paths to reach any given destination based on the traffic density, obstructions and accidents on the roads, and average speed of vehicles in a given time-frame. UAVs are leveraged to make several tasks, such as detecting accidents and congestion, collecting traffic information, and communicating with 5G BSs to reduce their communication burden (i.e., preventing vehicles in range to communicate directly with $5 \mathrm{G} \mathrm{BSs).} \mathrm{All} \mathrm{this}$ collected information is reported to the SDN controller to build an accurate view of traffic in real-time in each road segment. We assume that the SDN controller updates each vehicle with the current road conditions and generates re-planning to look for alternative shorter time paths in case of congestion or unexpected events blocking the roads. The various experiments conducted to evaluate the performance of this architecture show that it is possible to effectively adjust the travel path, and thus address the problem of static path planning.

The remaining of this paper is structured as follows. Section II provides an overview of the existing SDN-based traffic management solutions and route-planning algorithms in urban areas. Section III describes the proposed architecture and its major components. In Section IV, we discuss the experimental performance evaluation of our proposed algorithm. Finally, in Section V, we present the conclusion and future perspectives.

\section{RELATED WORKS}

$5 \mathrm{G}$ and beyond $5 \mathrm{G}(\mathrm{B} 5 \mathrm{G})$ are (at the time of writing this paper) the latest cellular networking technologies being developed, which are specifically designed to both provide high data rates and promise a latency of $1 \mathrm{~ms}$ for real-time applications [13]. These technologies support many technologies, including Heterogeneous Networks and SDN, to achieve massive device connectivity and ultra-low end-to-end latency [14]. Motivated by the features of $5 \mathrm{G}$ and $\mathrm{B} 5 \mathrm{G}$, we expect that network virtualization in a VANET environment exploited by SDN technology can provide multiple advantages and better performances to make commuting a pleasant experience [15]. Moreover, the integration of SDN technology into VANETs both helps to fulfill their management necessities and enhances their performances in terms of flexibility and programmability [16]. Even more, the SDN technology is expected to be a keystone for future intelligentization in a $6 \mathrm{G}$ [17]. In the following, we describe some background information and terminologies on $5 \mathrm{G}$ and its different technologies in the context of vehicular networking and their assistance by UAVs. Also, we discuss some route planning algorithms to set up the context of the problem.

By looking into the related research works, we only found a limited number of recent contributions in traffic management based on 5G, SDN, or VANET technologies. For instance, Ge et al. [18] proposed a hierarchical SDN-enabled vehicular architecture to address the issue of connectivity loss between the controller and switches while achieving the minimum transmission delay between vehicles. Liu et al. [19] leveraged the technologies of SDN, Mobile Edge Computing (MEC), and $5 \mathrm{G}$ to create a distributed architecture for urban traffic management. To quickly assign appropriate channels in an SDN-based IoT network, Tang et al. [20] considered a deep learning method and Partially Overlapping Channel Assignment (POCA) technique to efficiently avoid congestion and predict network traffic. In [21], a 5G-enabled SDN architecture is proposed, which provides larger bandwidth and higher data rates to enhance the capabilities of VANETs. Garg et al. [22] aimed to both maintain a certain level of quality of service (QoS) and ensure secure communication to VANETs by developing an SDN-based framework for $5 \mathrm{G}$ vehicular network. In [23], a novel SDN-based architecture for vehicle communication. It aims to minimize the communication cost between vehicles.

On the side of UAVs, we have witnessed an unexpected spread of UAV-based applications, spanning from monitoring, goods delivery, and public services, to communications, network assistance, and security [29]. Due to shorter network coverage in $5 \mathrm{G}$, UAVs can play the role of aerial BSs. For instance, in [30], UAVs are integrated to operate alongside 5G cellular networks. The work in [31] discussed the assistance of UAVs to $5 \mathrm{G}$ network supporting lightweight virtualization and network slicing. Sharma et al. [32] designed a novel approach to enhance $5 \mathrm{G}$ communication 
TABLE I: Feature comparison of path planning schemes.

\begin{tabular}{|c|c|c|c|c|c|c|c|}
\hline Features & Ref. [24] & Ref. $[25]$ & Ref. 26$]$ & Ref. 12] & Ref. [27] & Ref. $[\overline{28]}$ & Our application \\
\hline Basic ideology & $\begin{array}{c}\text { A balance } \\
\text { function between } \\
\text { distance and } \\
\text { time }\end{array}$ & $\begin{array}{l}\text { Big data analysis } \\
\text { for route } \\
\text { planning }\end{array}$ & $\begin{array}{l}\text { Route planning } \\
\text { with traffic } \\
\text { congestion and } \\
\text { oil consumption }\end{array}$ & $\begin{array}{l}\text { Leveraging both } \\
\text { current and } \\
\text { future traffic } \\
\text { status }\end{array}$ & $\begin{array}{l}\text { Comfort-based } \\
\text { route planning }\end{array}$ & $\begin{array}{l}\mathrm{V} 2 \mathrm{X} \text { and } \\
\text { driverless } \\
\text { technologies for } \\
\text { valet parking } \\
\text { technology }\end{array}$ & $\begin{array}{l}\text { Dynamic path } \\
\text { planning with } \\
\text { congestion } \\
\text { awareness }\end{array}$ \\
\hline Traffic view & Global & Global & Global & Global & Critical areas & Local & Global \\
\hline Route planning & Deterministic & Deterministic & Deterministic & Non-deterministic & Deterministic & Deterministic & Non-deterministic \\
\hline Re-routing & $x$ & $x$ & $\times$ & $\sqrt{ }$ & $x$ & $x$ & $\sqrt{ }$ \\
\hline Traffic incident awareness & $x$ & $x$ & $\times$ & $x$ & $\times$ & $x$ & $\sqrt{ }$ \\
\hline Major advantage & $\begin{array}{l}\text { Optimizing } \\
\text { parking lot path } \\
\text { planning }\end{array}$ & $\begin{array}{c}\text { Promoting } \\
\text { information } \\
\text { sharing between } \\
\text { Vehicles }\end{array}$ & $\begin{array}{l}\text { Decreasing oil } \\
\text { consumption }\end{array}$ & $\begin{array}{c}\text { Reducing } \\
\text { commuting time }\end{array}$ & $\begin{array}{l}\text { Demonstrating } \\
\text { comfortable } \\
\text { routes }\end{array}$ & $\begin{array}{l}\text { Selecting the } \\
\text { fastest route for } \\
\text { parking space }\end{array}$ & $\begin{array}{l}\text { Establishing the } \\
\text { shortest time } \\
\text { path in urban } \\
\text { areas }\end{array}$ \\
\hline Major Limitation & $\begin{array}{c}\text { Does not } \\
\text { consider } \\
\text { unexpected } \\
\text { events }\end{array}$ & $\begin{array}{l}\text { Neglecting the } \\
\text { future congestion } \\
\text { of selected paths }\end{array}$ & $\begin{array}{l}\text { Space and time } \\
\text { complexity of } \\
\text { the algorithm }\end{array}$ & $\begin{array}{l}\text { Unrealistic } \\
\text { gathered data }\end{array}$ & $\begin{array}{l}\text { Neglecting } \\
\text { drivers' feedback } \\
\text { among the } \\
\text { considered costs }\end{array}$ & $\begin{array}{c}\text { Neglecting } \\
\text { dynamic factors, } \\
\text { such as moving } \\
\text { obstacles }\end{array}$ & $\begin{array}{l}\text { The cost of } \\
\text { aerial and } \\
\text { terrestrial BSs' } \\
\text { deployment }\end{array}$ \\
\hline
\end{tabular}

networks by accurately deploying multiple UAVs that play the role of base stations. The authors in [33] proposed a UAV-assisted backhaul link considering the dynamic blockage of mmWave links. Different researches have involved vehicles to communicate with different entities based on the concept of Vehicle-to-Everything (V2X), which paves the path to UAVs to be fully integrated with VANETs on the ground [34]. As an example, Oubbati et al. in [35] proposed UAV-assisted VANET for traffic management and emergency vehicle guidance.

There are several route-planning strategies and algorithms proposed in the literature. The work of Xiaoxue et al. [24] proposed a path planning strategy based on a tradeoff between the path length and time balance to carry out optimal path planning. In [25], the authors proposed an edge-based big data analysis architecture to gather vehicle information and traffic status to provide the optimal driving path to vehicles. Zhang et al. [26] designed a route-planning algorithm based on real-time data, such as fuel consumption, road patterns, and feedback of drivers. In [12], a double rewarded value iteration network (VIN) for route planning and to predict how traffic will look like in the future by learning the routing decisions of experienced drivers. Yu et al. [28] proposed a route planning strategy to reach any parking spaces with the shortest time. This strategy can significantly help to minimize the congestion of the automatic valet parking lot. Based on the Dijkstra's algorithm, Li et al. [27] designed a cloud-aided route planner to detect anomalies on roads, and then consider the most comfortable route. For this purpose, three metrics are considered during the path planning process: (i) road roughness, (ii) travel time, and (iii) road remove.

Most of route planning schemes do not take into account unexpected events occurred on roads, and they just use the shortest distance or least time cost as the main functionality. Therefore, vehicles might be blocked at any time, and their paths cannot be dynamically adjusted when the predetermined paths become obstructed. In this work, we propose to enhance commuting in urban areas through an SDN-based route planning scheme applied over road networks that are weighted by accurate travel times of road segments. This scheme aims to dynamically identify shorter time paths while avoiding congestion and blocked roads. In addition, our second aim is to meet a certain level of networking requirements while enhancing the exploitation of existing network infrastructures.

To summarize, Table I provides a comparative study between our proposed scheme and the previously proposed route planning approaches based on several features.

\section{AN SDN-ENABLED APPROACH FOR VEHICLE PATH-PLANNING (SEARCH)}

Nowadays, driving a vehicle in a big city become almost unbearable due to the enormous traffic jam. As discussed above, various emerging technologies are seen as promising solutions in reducing traffic congestion on roads and providing better monitoring of traffic conditions. Therefore, we need to design a new architecture based on these technologies to improve emergency responsiveness and save time in transporting people, while considering the access, connectivity, and analysis features. In this section, we present this architecture and its main components. Moreover, we describe operation details of this framework, including packet exchange policy and vehicle traffic calculation. Finally, we discuss a specific use case when a vehicle wants to reach a given target destination and how our path planning algorithm deals with it.

\section{A. Network Model}

As depicted in Fig. 1, the overall network architecture is a combination of different nodes, such as vehicles, UAVs, and $5 \mathrm{G}$ base stations (5G BSs). The organization of the network is based on SDN technology. SDN controllers are mainly responsible for managing this technology by collecting data from all network nodes for building a global traffic information graph of all vehicles on the roads. For this purpose, all vehicles periodically send Hello packets to the SDN controllers through BSs and UAVs that are on the front line of this data collection. The SDN controllers are primarily in charge of updating its neighboring list of each vehicle in the light of the latest information received from periodically Hello packets sent by vehicles to estimate the number of vehicles and their fluidity in each road segment. In particular, the location, speed, and direction of vehicles are essential traffic information on roads, which can be estimated by several wireless location technologies other than GPS in large urban cities where skyscrapers and other obstructions may block GPS signals. 


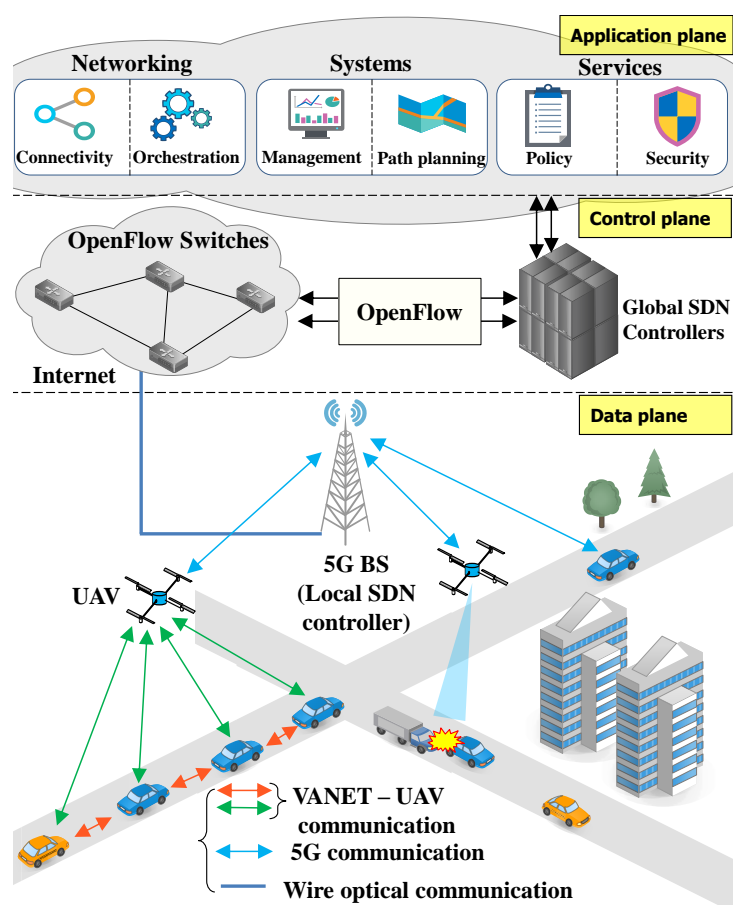

Fig. 1: The three-tier architecture of SEARCH.

All this collected information allows vehicles to make smart and efficient decisions regarding the path planning process.

1) Modes of communications: Our network architecture is based on three modes of communications, namely Vehicle-to-BS (V2BS), Vehicle-to-UAV (V2U), and Vehicle-to-Vehicle (V2V). In V2U communication, UAVs can communicate directly with vehicles based on the assumption that they will not be affected by obstructions when they are in range of each other. Also, they can act as collector nodes gathering periodically all data related to vehicles wherever it is possible. V2BS are used for control message dissemination, including also the periodical gathering of vehicles' information (i.e., position, speed, and direction) included in their Hello packets, either from vehicles or from UAVs. It should be stressed that UAVs communicate with vehicles within the UAVs' coverage area, while the BSs communicate with vehicles that are out of the coverage area of any UAVs. In other words, UAVs have top priority to collect traffic information on roads when they are hovering any areas in order to reduce the network load on 5G BSs. Furthermore, each BS monitors the traffic information in a real-time manner and follows each vehicle requesting a path planning by sending essential updates about the status of the traffic to all vehicles in its vicinity. In V2V communication, vehicles can communicate with each other or serve as relays, which does not enter the scope of the paper.

2) SDN planes: The network architecture is divided into three SDN planes that are assigned specific SDN responsibilities and rules. We describe each SDN plane below:

- Control plane: is managed by the SDN controllers that define a set of rules with OpenFlow protocol. These rules determine how the interconnection units $(e . g ., 5 \mathrm{G}$ BSs, UAVs, and vehicles) transmit the data frames to the upper planes through optical fiber links. Generally, the controllers monitor the positions of all vehicles in the area in order to allow vehicles to plan their shorter time paths to reach their target destinations quickly.

- Application plane: includes several software modules (i.e., applications) related to different aspects, such as security applications, traffic efficiency, networking, and entertainment. The mode of how to provide open interfaces to such modules is considered as an advantageous factor to make simpler the network optimization of functions and be up-to-date with the new technology developments.

- Data plane: consists of the physical devices and infrastructures in the network. All these physical resources play the role of OpenFlow switches, and they are mainly including vehicles, UAVs, 5G BSs. Each vehicle can generate various data information, such as speed, position, previous routes, etc. Equipped with required communication modules, vehicles can transmit all these generated data to the controller either through cellular BSs or UAVs.

To further clarify the functioning of our SDN-based architecture, we show the key functionalities of each considered component in Fig. 2 .

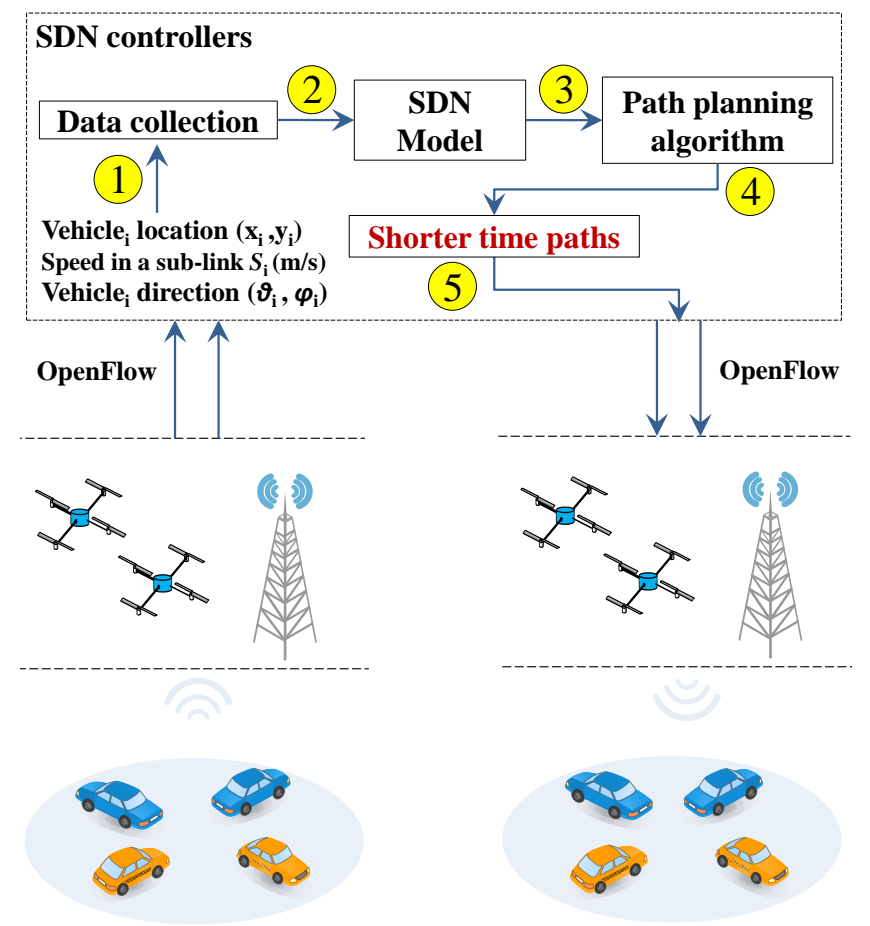

Fig. 2: Key functionalities and components of SEARCH.

3) SDN components: The working of the SDN model components is as follows:

- SDN Controllers: they belong to the control plane, and they have control of the overall wired and wireless networks, including 5G BSs, UAVs, and vehicles. Moreover, they are considered as intermediate nodes between the data and application planes. 
- 5G BS: it can act as a router and at the same time as an SDN switch, which is responsible for ensuring the control of different network devices, such as UAVs and vehicles in a particular area. Besides, it can estimate the traffic fluidity on road segments that are not covered by UAVs.

- UAVs: they act also as SDN switches and they are in charge of estimating the traffic fluidity information on roads and forwarding this information to the closest $5 \mathrm{G}$ BSs.

- Vehicles: they are equipped with two on-board network devices to support the communication with both UAVs and $5 \mathrm{G}$ BSs.

It is worthy to summarize the role of the major components exploited by SEARCH in its processes in TABLE II]

TABLE II: The role and advantages of $5 \mathrm{G}, \mathrm{SDN}$, and UAVs over SEARCH.

\begin{tabular}{|l|l|l|l|}
\cline { 2 - 4 } \multicolumn{1}{c|}{} & 5G BSs & UAVs & SDN \\
\hline $\begin{array}{l}\text { Path } \\
\text { planning }\end{array}$ & $\begin{array}{l}\text { Updating } \\
\text { vehicles }\end{array}$ & Traffic gathering & $\begin{array}{l}\text { Estimating } \\
\text { shorter time } \\
\text { paths }\end{array}$ \\
\hline Accidents \& & $\begin{array}{l}\text { Warning vehicles } \\
\text { before or at } \\
\text { intersections }\end{array}$ & Detection & $\begin{array}{l}\text { Updating path } \\
\text { planning }\end{array}$ \\
\hline Nraffic status & \multicolumn{2}{|c|}{ Collecting traffic information } & $\begin{array}{l}\text { Estimating } \\
\text { density of } \\
\text { vehicles at road } \\
\text { segments }\end{array}$ \\
\hline Networking & $\begin{array}{l}\text { Providing } \\
\text { bandwidths and } \\
\text { scalability }\end{array}$ & $\begin{array}{l}\text { Reduce } \\
\text { communication } \\
\text { burden over 5G } \\
\text { BSs }\end{array}$ & $\begin{array}{l}\text { Managing } \\
\text { network } \\
\text { infrastructures } \\
\text { and fully } \\
\text { exploiting their } \\
\text { resources }\end{array}$ \\
\hline
\end{tabular}

\section{B. Assumptions}

The SEARCH framework assumes that the SDN controllers have full access to the digital map to locate neighboring path junctions, as well as the required road information. Each road segment is partitioned into equal size zones of $30 \mathrm{~m}$ each (c.f., Fig. 3). This partition is based on the assumption that the traffic state is considered to be homogeneous, where strict lane discipline is followed by all vehicles. UAVs and 5G BSs are considered to cover at least one zone where they collect data from vehicles and maintain a local view of the traffic state of their controlled zones. Each zone is supposed to be controlled by at least one BS, a UAV, or both. A global view of the overall traffic state can be obtained by exchanging the sectional view of adjacent BSs or UAVs with the SDN controllers.

The connectivity between vehicles and UAVs is ensured via IEEE 802.11p wireless interfaces. Moreover, vehicles and UAVs can also communicate with 5G BSs through appropriate cellular interfaces. The connectivity between $5 \mathrm{G}$ BSs and SDN controllers is ensured through high-speed optical fiber links. In the case when UAVs are hovering in clear weather, they can detect incidents on roads using their image capture and processing capabilities. Otherwise, UAVs can exploit V2U communication to report such events from the involved terrestrial nodes. It is worthy to note that these two solutions hold applicable for SEARCH. Since the satellite signals might be blocked by skyscrapers, SEARCH assumes that

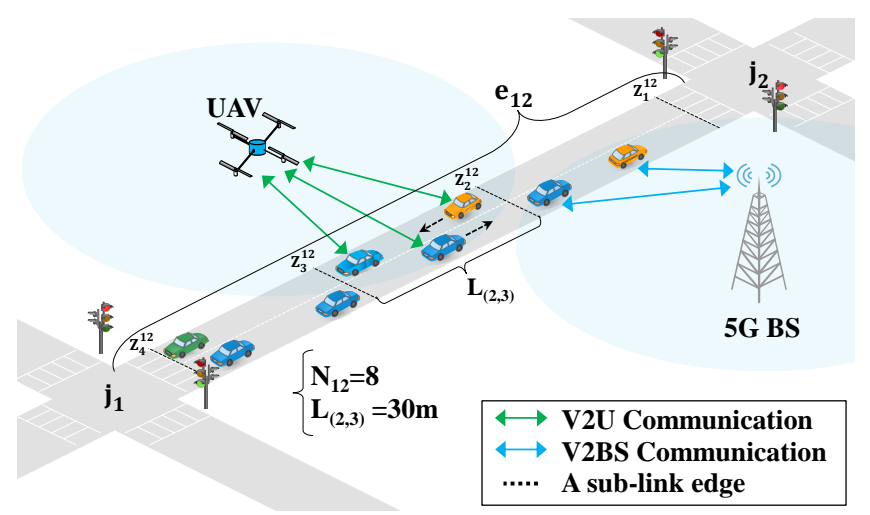

Fig. 3: Travel time estimation.

the localization service of vehicles is based on the direction of arrival (DoA)/time of arrival (ToA) [36]. This type of localization aims to realize GPS-free localization based on device-to-device (D2D) communications or 5G antenna. The accident detection process, the vehicle localization service, and the energy consumption of UAVs are our future research works and are out of the scope of the present study.

\section{Functionality of SEARCH}

In any path planning strategy, the SDN technology can be considered as the best solution to cover any areas efficiently, collect, analyze, and transmit crucial information related to any events that occurred on roads. Our framework is designed to allow 5G BSs and UAVs to sense the variation of traffic status on the surrounding road segments. Also, it enables $5 \mathrm{G}$ BSs to collect all possible data from vehicles and UAVs. All gathered data are transmitted to the SDN controllers to allow them to both build a global view of the traffic status on the roads and quickly establish shorter time paths between any pairs of locations.

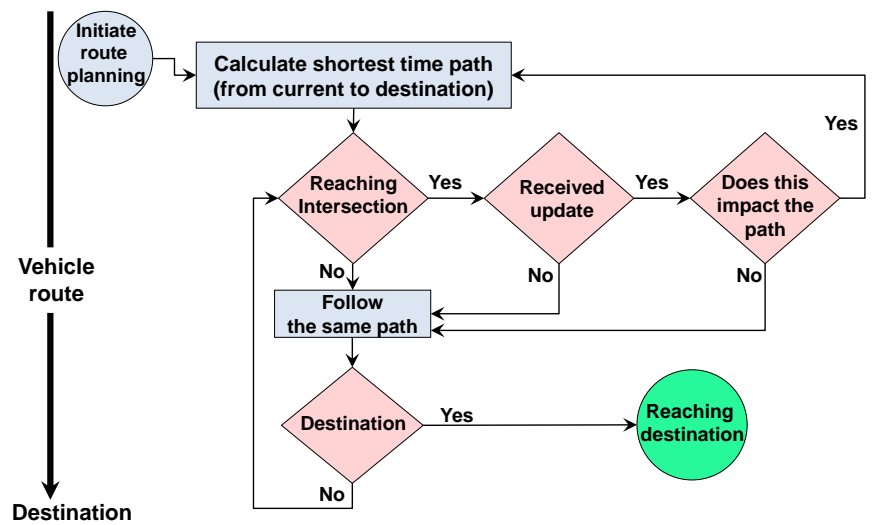

Fig. 4: Flowchart illustrating the functionality of SEARCH.

When a vehicle wants to reach a given destination, the SDN controllers are already supposed to have the current global vision of all surrounding road segments. Initially, the controllers calculate the shortest time path from the current location to the desired destination of the vehicle, according to the Dijkstra algorithm. Whenever the vehicle reaches 
an intersection, it receives an update related to the traffic conditions. If this update affects the earliest established path, the Dijkstra algorithm is re-applied to calculate a new shortest time path. Otherwise, the vehicle continues following the same path. This process is repeated until the vehicle reaches its desired destination. For more clarification, we summarize these steps in the flowchart depicted in Fig. 4 . In the remainder of this section, we first describe the vehicle traffic calculation and then present the application of our SEARCH routing planning framework on a concrete example.

1) Journey time calculation: Vehicle driving time is considered as an essential criterion of information to define the minimum cost routes in the path planning process. Moreover, it is also exploited as data to support driver decisions through on-board guidance systems, editable message boards, and radio dissemination. In our case, the road network is modeled as a cyclic graph $G=(J, E)$ to provide flexibility to use well-known techniques in graph theories. $J$ is defined by the set of $N$ nodes or vertices (i.e., junctions), where $J=\left\{j_{i}\right\}_{i=1}^{N}$. $E$ is a set of edges between vertices (i.e., directional links between junctions), where $E=\left\{e_{i j}=\left(j_{i}, j_{j}\right), j_{i}, j_{j} \in J\right\}$ and $e_{i j} \neq e_{j i}$. Since the edges are directed, the traffic in both directions could be either dependent or independent, according to the presence of traffic signals at intersections, where SEARCH operates correctly in both situations (c.f., Fig. 3). In Table [II], we define a set of parameters that are useful to describe the model clearly.

TABLE III: Summary of parameters of SEARCH.

\begin{tabular}{|l|l|}
\hline Parameter & Definition \\
\hline$e_{i j}$ & A link from $j_{i}$ to $j_{j}$. \\
\hline$D_{i j}$ & Distance between $j_{i}$ and $j_{j}$. \\
\hline$S_{i j}$ & Default limited speed of $e_{i j}$. \\
\hline$T_{i j}$ & Journey time of $e_{i j}$, where $T_{i j}=\frac{D_{i j}}{S_{i j}}$. \\
\hline$Z_{k}^{i j}$ & Beginning of the $k^{t h}$ sub-link in $e_{i j}$. \\
\hline$N_{i j}$ & Number of zones in $e_{i j}$. \\
\hline$j_{k}$ & $k^{t h}$ junction. \\
\hline$L_{(k, k+1)}$ & Length between $Z_{k}^{i j}$ and $Z_{k+1}^{i j}$ (by default $30 \mathrm{~m}$ ). \\
\hline
\end{tabular}

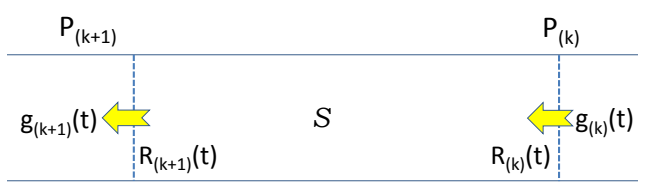

Fig. 5: Journey time in a sub-link.

To estimate the full journey time of a given link, we need to calculate the journey time in each sub-link belonging to the link $e_{i j}$, such as:

$$
T_{i j}=\sum_{k=1}^{N} t_{k}^{i j}
$$

Where $T_{i j}$ is the journey time of the whole link $e_{i j} . t_{k}^{i j}$ is the journey time of each sub-link constituting $e_{i j}$. To calculate $t_{k}^{i j}$, the journey time of the sub-link $\left(Z_{k}^{i j}, Z_{k+1}^{i j}\right)$ can be estimated using a combination between the traffic information gathered by UAVs and 5G BSs and the Macroscopic Model [37]. Therefore, we model the relationship between density and traffic flow of a sub-link as a continuous fluid model, as depicted in Fig. 5

Based on this modeling, we can then calculate the average speed, and thus the journey time in the sub-link based on these two parameters. The Macroscopic Model is defined as follows:

$$
\frac{\partial}{\partial t} R(P, t)=\frac{\partial}{\partial P} g(P, t)
$$

Where $R$ is the vehicle density by distance. $g$ is the traffic flow per time, while $P$ and $t$ are defined as the location and the current time, respectively. The average speed in the sub-link can be calculated based on the equation (2) as follows:

$$
S=\frac{g}{R}
$$

Based on the equation (3), the journey time of the sub-link $\left(Z_{k}^{i j}, Z_{k+1}^{i j}\right)$ can be estimated as follows:

$t_{k}^{i j}=\frac{L_{(k, k+1)}}{S}=\frac{L_{(k, k+1)}}{g} \times R=\frac{L_{(k, k+1)} \times\left\{R_{(k+1)}+R_{(k)}\right\}}{\left\{g_{(k+1)}-g_{(k)}\right\}}$

Where $L_{(k, k+1)}$ is the length of each sub-link (i.e., $30 \mathrm{~m}$ ) depending on the length of the road segment where $L_{(k, k+1)}$ can be sometimes less or more than $30 \mathrm{~m} . g_{(k)}$ is the gathered traffic at the location $P_{(k)}$ at time $t . R_{(k)}$ is the density of node at the location $P_{(k)}$ at time $t$. Therefore, we can calculate the traffic throughout the sub-link (i.e., gathered traffic in $Z_{k+1}^{i j}$ ) based on the following equation (5):

$$
g_{k+1}^{i j}(t)=\frac{\left(n_{k+1}^{i j}-n_{k}^{i j}\right)+X}{t}
$$

Where $X$ is the total number of vehicles residing in the sub-link between $Z_{k}^{i j}$ and $Z_{k+1}^{i j}$ before the traffic gathering. As for $n_{k}^{i j}$ and $n_{k+1}^{i j}$, they represent the number of vehicles entering and leaving the sub-link between $Z_{k}^{i j}$ and $Z_{k+1}^{i j}$, respectively. Similarly, the density of vehicles in the sub-link can be computed as follows:

$$
R_{k+1}^{i j}(t)=\frac{\left(n_{k+1}^{i j}-n_{k}^{i j}\right)+X}{L_{(k+1, k)}}
$$

According to the equation (3), we can deduct the average speed in the sub-link between $Z_{k}^{i j}$ and $Z_{k+1}^{i j}$ as follows:

$$
S_{k+1}^{i j}=\frac{g_{k+1}^{i j}}{R_{k+1}^{i j}}=\frac{\left(n_{k+1}^{i j}-n_{k}^{i j}\right)+X}{t} \times \frac{L_{(k+1, k)}}{\left(n_{k+1}^{i j}-n_{k}^{i j}\right)+X}
$$

Finally, the journey time in the sub-link between $Z_{k}^{i j}$ and $Z_{k+1}^{i j}$ in the link $e_{i j}$ can be easily extracted based on the formula (4) as follows:

$$
t_{k}^{i j}=\frac{L_{(k, k-1)}}{S_{k}^{i j}}
$$


2) Case Study: SEARCH route planning: Arriving on time is considered as a crucial factor in most path planning strategies. This punctuality raises two key questions: "When should I start the car?" and while on the way "Which path should I follow?". Indeed, the majority of path planning strategies are deterministic, which can be used to solve the problem. Nevertheless, practically, the majority of these strategies focus on minimizing the journey distance and time well before starting the travel without considering unexpected events or congestion that would be happened on the road. Consequently, the proposed framework is based on a non-deterministic approach to change this impasse, as shown in Fig. 6 With the assistance of a low-latency SDN-based heterogeneous network, the novel path planning scheme would be enhanced by two significant measures: (i) Congestion and accident detection and (ii) shorter time paths calculation.

Congestion and incident detection: In the process of traditional path planning, when a vehicle requests to reach a target destination, the centralized architecture tries to compute the full path based on one or several features, such as the distance, the journey time, the oil consumption, etc. However, the major limitation of such architectures is that there is no continual and real-time assessment of the variable nature of traffic congestion on the roads and the unexpected incidents that may occur during the journey of the vehicle to avoid an indefinite duration of the trip. In the SEARCH framework, the

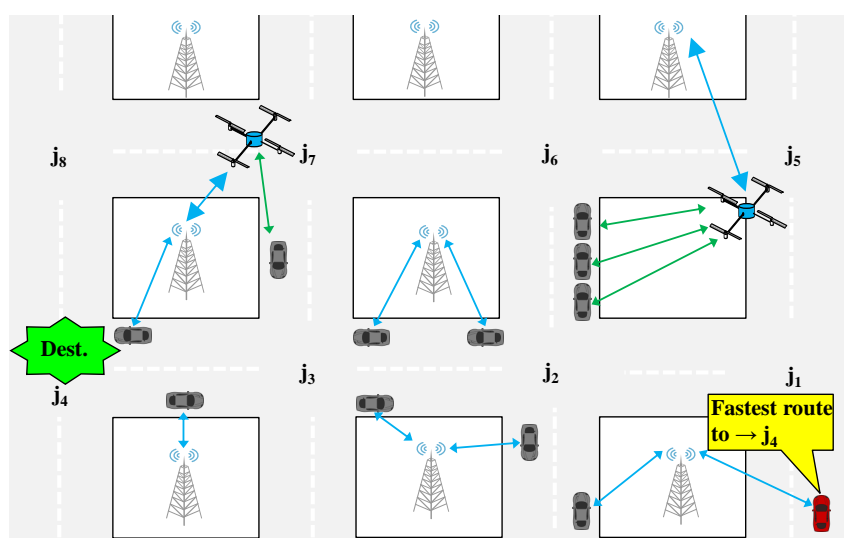

(a) Phase 1 .

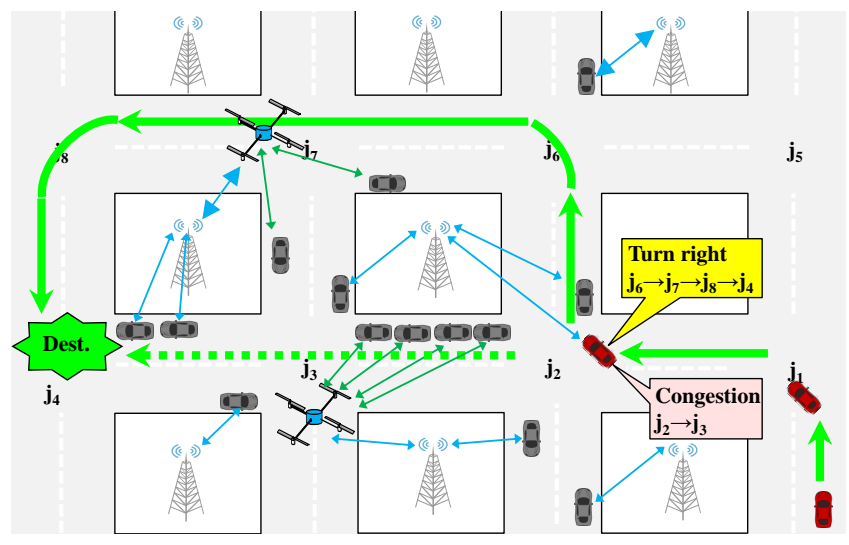

(c) Phase 3. traffic status of vehicles is permanently collected by $5 \mathrm{G}$ BSs and UAVs and forwarded to the SDN controllers. Besides, the hovering UAVs in the sky can make a real-time video diagnosis of any events on the roads that can hinder or block the traffic. All these events' information is also gathered by SDN controllers for analysis.

As illustrated in Fig. 6(a), since there are no UAVs in range, the red vehicle requests the closest $5 \mathrm{G}$ BS to get the shortest time path towards the junction $j_{4}$ (i.e., the target destination). Once the request is fulfilled, the red vehicle starts its journey through the path $j_{1} \rightarrow j_{2} \rightarrow j_{3} \rightarrow j_{4}$ (c.f., Fig. 6(b)). However, the journey will not last long, until a crucial update impacting the original path is intercepted at junction $j_{2}$. This update informs the red vehicle that the segment between $j_{2}$ and $j_{3}$ becomes congested. This requires initiating the path planning calculation from the current junction $j_{2}$. This calculation results in obtaining an alternative shorter time journey through the path $j_{2} \rightarrow j_{6} \rightarrow j_{7} \rightarrow j_{8} \rightarrow j_{4}$. At the junction $j_{7}$, the red vehicle receives another crucial update from the closest BS, which represents an accident that occurred between $j_{7}$ and $j_{8}$ (see Fig. 6(d)). Therefore, re-initiating the calculation of the shortest time path from $j_{7}$ is unavoidable. Another journey is obtained from the current junction $j_{7}$ through the path $j_{7} \rightarrow j_{3} \rightarrow j_{4}$, until reaching its target destination. This process is summarized in TABLE IV in which there are the estimated journey times for each path.

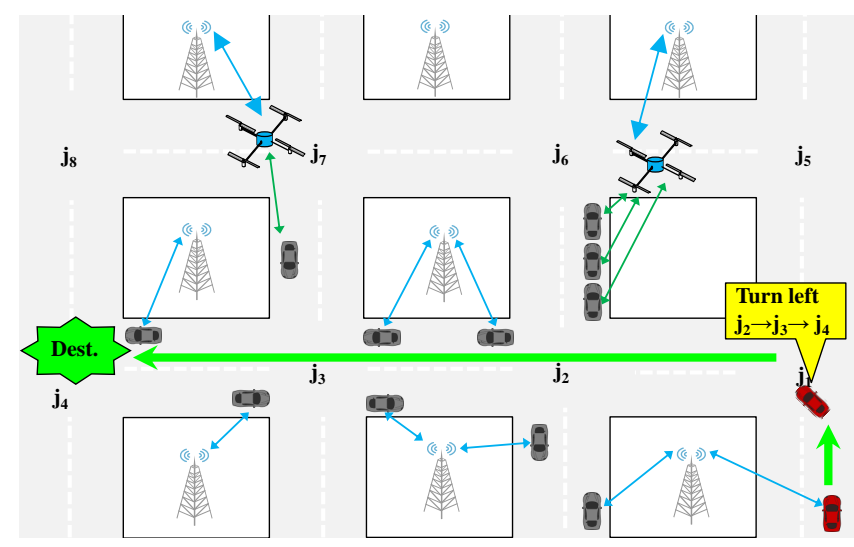

(b) Phase 2.

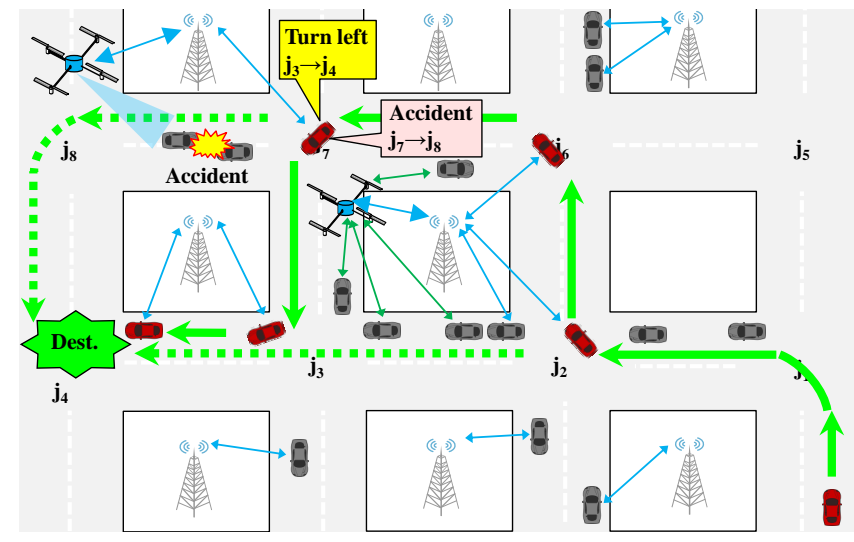

(d) Phase 4.

Fig. 6: A map processing test of SEARCH. 
TABLE IV: Processing and Functionality of SEARCH.

\begin{tabular}{|c|c|c|c|c|c|}
\hline Source & Destination & Journey path(s) & $\begin{array}{l}\text { Estimated journey time (Dijkstra } \\
\text { cost) from the current junction }\end{array}$ & $\begin{array}{l}\text { Update impacting the } \\
\text { path }\end{array}$ & Cause of the update \\
\hline \multirow{3}{*}{$j_{1}$} & \multirow{3}{*}{$j_{4}$} & $j_{1} \rightarrow j_{2} \rightarrow j_{3} \rightarrow j_{4}$ & $115 \mathrm{~s}$ & Occurred at $j_{2}$ & Congestion \\
\hline & & $\ldots \rightarrow j_{2} \rightarrow j_{6} \rightarrow j_{7} \rightarrow j_{8} \rightarrow j_{4}$ & $100 \mathrm{~s}$ & Occurred at $j_{7}$ & Accident \\
\hline & & $\ldots \rightarrow j_{7} \rightarrow j_{3} \rightarrow j_{4}$ & $60 \mathrm{~s}$ & Not occurred & - \\
\hline
\end{tabular}

Dynamic Shorter time paths calculation: The SDN controllers permanently calculate the journey times in each lane of road segments. After that, the journey times are shared with requesting vehicles. The Dijkstra algorithm is applied to calculate shorter time paths. For this purpose, this algorithm is enhanced to consider only the influence of journey time in each link and to find the optimal path. Consequently, the optimal path costs the least time, where all weights (i.e., journey times) must be non-negative.

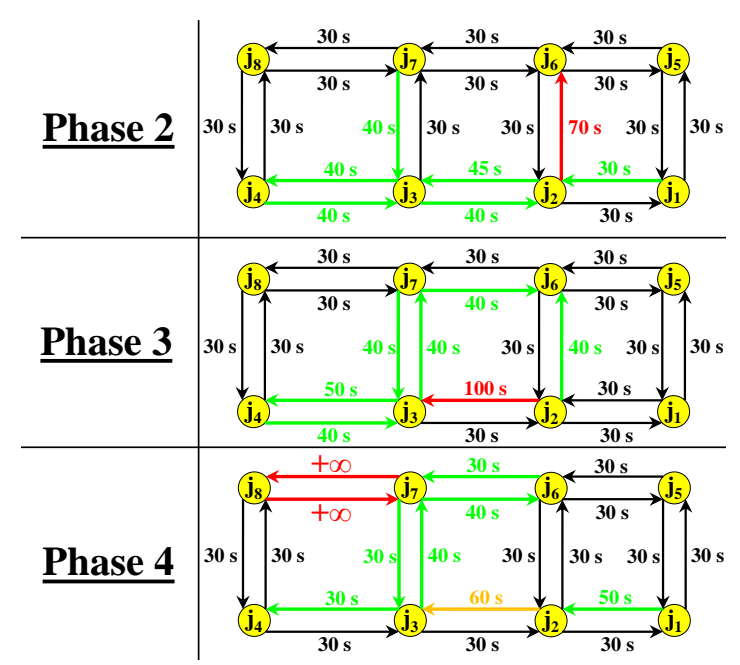

Fig. 7: Weighted directed graphs of Fig. 6

In our case, our framework transforms the map shown in Fig. 6 into a directed weighted graph consisting of nodes and edges, as illustrated in Fig. 7 Given that we have considered a map with the same length of road segments, we distinguish that all empty lanes take at most 30 seconds to be crossed. The other lanes can be crossed, depending on the circulation of vehicles inside them. At each update impacting the established path, a new calculation of the shortest time path is made. For instance, the red vehicle has carried out two different updates at the junctions $j_{2}$ and $j_{7}$ to avoid congested or blocked road segments. Indeed, at the first step, the red vehicle computes the journey time at $j_{1}$, which is estimated at $115 \mathrm{~s}$. However, after traveling 30 seconds, the vehicle reaches the junction $j_{2}$, in which it receives a crucial update impacting the current path. Therefore, the vehicle re-calculates the shortest journey time at $j_{2}$ to find a new shorter time path through $j_{2} \rightarrow j_{6} \rightarrow j_{7} \rightarrow j_{8} \rightarrow j_{4}$, which is estimated at 100 seconds instead of taking the previous path with an estimation of 150 seconds. After running 100 seconds, the red vehicle reaches the junction $j_{7}$ to find the road blocked in front of it (i.e., between $j_{7}$ and $j_{8}$ ). Therefore, a new shortest time alternative path is found through $j_{7} \rightarrow j_{3} \rightarrow j_{4}$, which is estimated at 60 seconds to finally reach the target destination. In the end, the red vehicle has crossed the full path towards $j_{4}$ in 160 seconds, which is the shortest time while considering the variation of traffic and unexpected events occurred on the roads. Algorithm 1 shows the process of congestion avoidance using SEARCH. Moreover, it also illustrates the procedure of calculating new routes when a crucial update is received. This process is permanently repeated to update vehicles within each intersection through UAVs or BSs.

\begin{tabular}{l}
\hline Algorithm 1: Congestion avoidance using SEARCH \\
\hline Input: $\quad / /$ A set of vehicles \\
$C=\left\{c_{i}\right\}_{i=1}^{N} ; \quad$ // Roads with attributes \\
$H:$ Hello packet between UAVs, 5G BSs, and vehicles. \\
$K=\left\{e_{i j}\right\}_{i, j=1}^{N} ; \quad / /$ A set of congested roads \\
$M=\emptyset ; \quad$ ind
\end{tabular}

Output:

$R$ : Shortest time route.

1 At each UAVs and 5G BSs

foreach $e_{i j} \in G$ do

$$
\mid \begin{aligned}
& \text { Collect }\left(c_{i} \text { location }\right) ; \\
& \text { positions } \\
& \text { Collect }\left(\left\{S_{n}^{i j}\right\}_{n=1}^{N}\right) ; \quad / / \text { Vehicles' } \\
& \text { sub-link } \\
& \text { Update }\left(e_{i j},\left\{S_{n}^{i j}\right\}_{n=1}^{N},\left\{c_{i}\right\}_{i=1}^{N} \in e_{i j}\right)
\end{aligned}
$$

2 Congestion roads detection

foreach $e_{i j} \in K$ do

$$
\begin{array}{rlrl}
\text { Calculate }\left(S^{i j}\right) ; & / / \text { Average speed in } e_{i j} \\
\text { if } S^{i j} & \leq 0.2 \text { then } & & \\
L M & \cup\left\{e_{i j}\right\} ; & & / / \text { Add } e_{i j} \text { into } M
\end{array}
$$

\section{At each intersection}

foreach $c_{i} \in C$ do

route $\leftarrow \operatorname{Extract}\left(c_{i} \cdot B\right) ; \quad / /$ Extract the

vehicle route from Hello packets

foreach $e_{i j} \in$ route do

$$
\begin{aligned}
& \text { if } e_{i j} \in M \text { then } \\
& \begin{array}{l}
K-\left\{e_{i j}\right\} ; \quad / / \text { Exclude the } \\
\text { congested road from } K \\
\text { Extract }\left(c_{i}\right. \text { location); // Get the } \\
\text { current location of } c_{i} \\
\text { foreach } U A V \text { and } B S \text { do } \\
\quad \begin{array}{l}
\text { Update }\left(K,\left\{e_{i j}, T_{i j}\right\}_{i, j=1}^{N}\right) ; / / \text { Update } \\
\text { road attributes }
\end{array} \\
\text { route } \leftarrow \text { Shortest time }(K) ; / / \text { Shortest } \\
\text { time path // Send the newly } \\
\text { Send(route, } \left.c_{i}\right) ; \quad \text { route to the vehicle } \\
\text { calculated routed }
\end{array}
\end{aligned}
$$




\section{Performance Evaluation}

To evaluate the effectiveness of our SEARCH framework, we conduct a set of experiments. We considered OMNeT++ 4.3 [38], which is complemented by SUMO [39] and MobiSim [40] as two mobility generators producing the random motions of vehicles and UAVs, respectively. At a first step, we import a test urban area from OpenStreetMap [41], which is known for its busy streets of vehicles (see Fig. 8). Secondly, we extract the road layout in the form of directed edges and nodes (i.e., blue lines and red circles in Fig. 8(b), respectively), we define the number of vehicles and UAVs for each scenario, and we select a number of accidents in each scenario of simulation. To summarize, in this section, we first present the simulation model and the different considered parameters. Finally, we provide a clear interpretation of the obtained results.

\section{A. Parameter Settings}

To evaluate the performances of our framework, we place a sufficient number of UAVs and $5 \mathrm{G}$ BSs such that we ensure a full coverage along the roads. This full-coverage allows us to get a global view of vehicular network topology by collecting the movement information of all vehicles. For the vehicular network, we consider IEEE $802.11 \mathrm{p}$ wireless interfaces for each vehicle and UAV using the framework Veins [42]. Also, we deploy up to 400 vehicles $/ \mathrm{km}^{2}$ and 144 UAVs, which are randomly distributed to simulate traffic and to capture any incidents on the roads. We randomly select a pair of starting and ending points located at different intersections (i.e., red circles), as shown in Fig. 8(b) It is worthy to note that each point in the results represents the mean of 10 simulation runs with a $95 \%$ confidence interval. The performance of our path planning algorithm is compared to traditional distance-based and time-based path planning mechanisms that calculate the full path to be traveled before the beginning of the journey. We also evaluate the performance of our SDN-based architecture under different metrics and compare it with both the architecture presented in [18] and Non-SDN-based architecture. Table $\mathrm{V}]$ summarizes the main simulation parameters used in our experiments.

TABLE V: Simulation parameters to evaluate SEARCH.

\begin{tabular}{ll}
\hline Parameters & Values \\
\hline Simulation time & $900(\mathrm{~s})$ \\
Area size & $2 \times 2 \mathrm{~km}^{2}$ \\
Normal traffic density & $\approx 80$ vehicles $/ \mathrm{km}^{2}$ \\
Rush-hour traffic density & $\approx 480$ vehicles $/ \mathrm{km}^{2}$ \\
Number of incidents & {$[5,30]$} \\
Number of UAVs & {$[16,144]$} \\
Number of 5G BSs & $1 \mathrm{BS} / \mathrm{km}$ \\
Vehicle speed $s_{\max }$ & $14 \mathrm{~m} / \mathrm{s}$ \\
\hline Channel frequency & $5.890 \mathrm{e} 9 \mathrm{~Hz}$ \\
Transmit power & $21.5 \mathrm{dBm}$ \\
Sensitivity & $-81.5 \mathrm{dBm}$ \\
Path loss model & Free-space \\
PHY/MAC model & IEEE $802.11 \mathrm{p}$ \\
Bit rate & $18 \mathrm{Mbit} / \mathrm{s}$ \\
\hline Coverage of UAVs and vehicles & $\approx 300 \mathrm{~m}$ \\
Hello interval & $0.1(\mathrm{~s})$ \\
Packet size & $1 \mathrm{~KB}$ \\
\hline
\end{tabular}

\section{B. Results Analysis}

To evaluate the performance of our framework and the other path planning algorithms, we first calculate the total journey time of the vehicle to reach the target destination for each applied path planning algorithm (c.f., Fig. 9). As depicted in Fig. 9(a), our framework portrays an

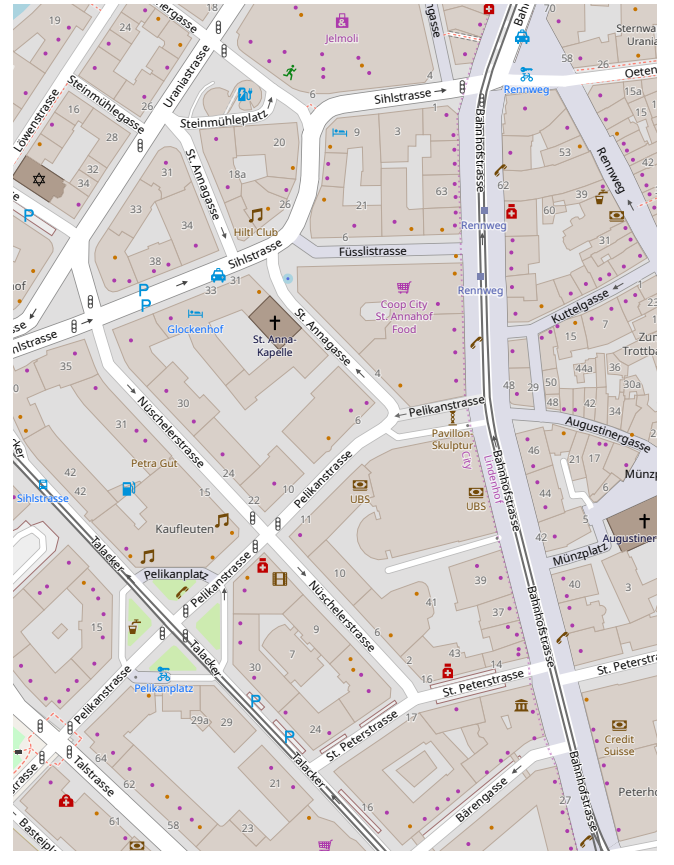

(a) Generated map.

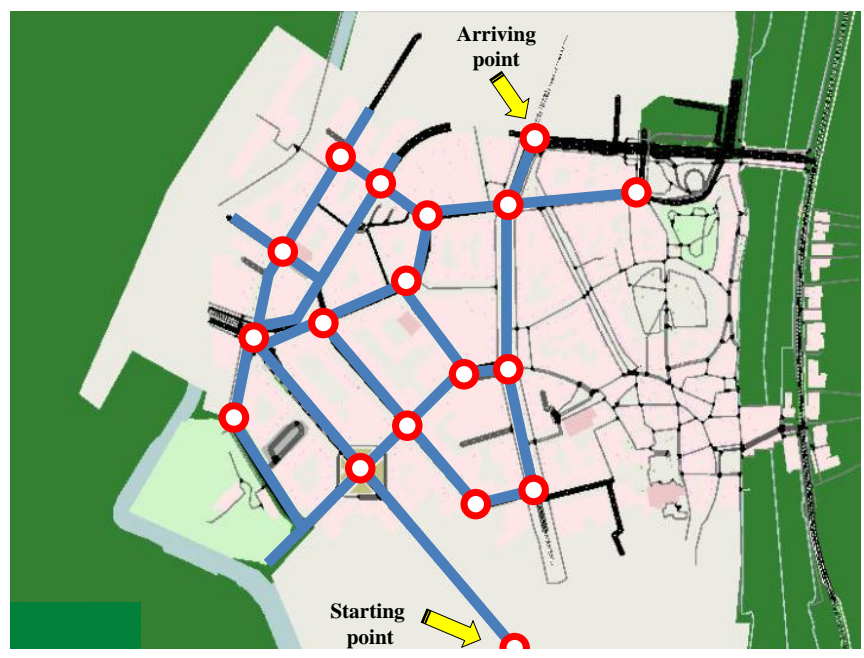

(b) Road layout.

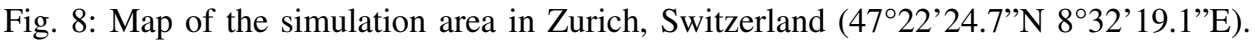




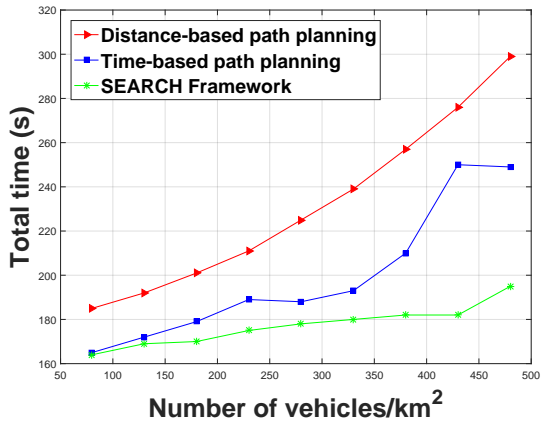

(a) Total time vs. Density of vehicles (UAV density $=16$, Number of incidents $=5$ ).

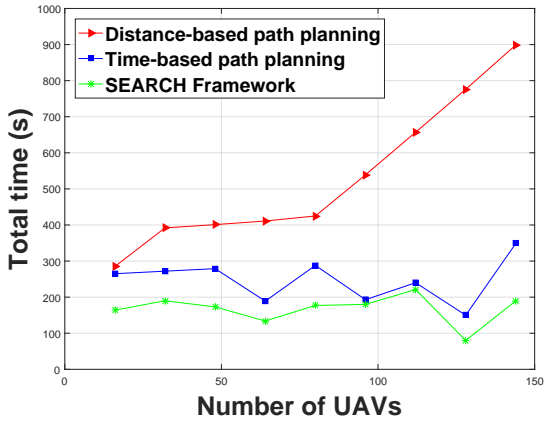

(b) Total time vs. Density of UAVs (Vehicle density $=280$, Number of incidents $=5$ ).

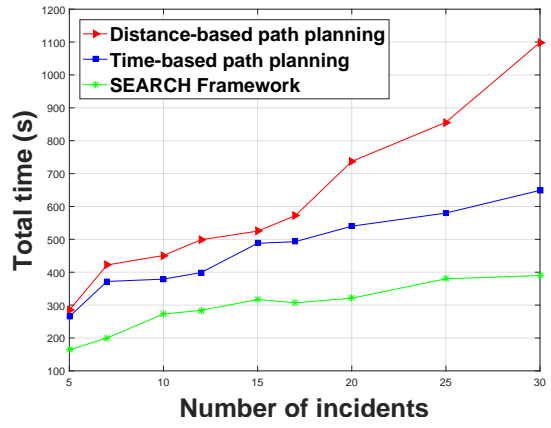

(c) Total time $v s$. Number of incidents (Vehicle density $=280$, UAV density=16).

Fig. 9: Simulation results of journey time of SEARCH.

outperforming performance under different vehicle densities. Indeed, compared to other algorithms, SEARCH can decrease the journey time by more than $20 \%$. This minimization is due to its dynamic nature to calculate shorter time paths while being aware of both incidents and traffic congestion on the roads. However, for the other algorithms, the average time journey tends to be increased as the density of vehicles increases. This can be explained by the continuous selection of the initial path, thus exposed to traffic congestion and incidents during the vehicle journey. In Fig. 9(b), we distinguish that SEARCH achieves the least journey time as the density of UAVs increases. This is because many road segments are controlled by the increasing number of UAVs and generate alerts to the SDN controllers at each detected incident. Nevertheless, the results of the other algorithms are not affected by the variation of UAV density since their functioning is based only on the density of vehicles and the length of paths. Fig. 9(c) reveals that with only 16 UAVs, SEARCH can enhance the vehicle journey time by detecting most of the incidents and effectively regulate the vehicle journey. In essence, compared to the other algorithms, SEARCH allows the vehicle to avoid the traffic jam that is formed around or on the way to the accident.

To compare the distance traveled for all the evaluated algorithms, we perform different experiments under different densities of vehicles and UAVs while varying the number of incidents. As a result, we obtain three graphs represented in Fig. 10. As shown in Fig. 10(a), SEARCH achieves a low traveled distance compared to time-based path planning. This is because SEARCH establishes congestion aware paths, thus avoiding traffic jams whenever it is possible and occasionally reducing distances. However, it is not the case for time-based path planning that initially selects the full path to the target destination, which should avoid all congested and blocked roads, and thus occasionally results in some peaks of traveled distances during the vehicle journey. It is worthy to note that even if we expect a proportional behavior of traveled distances according to the density of vehicles, but due to the time-based criteria of selecting paths in both SEARCH and time-based approach, Fig. 10(a) shows unstable behavior of traveled distances. As for Fig. 10(b), decrease of traveled distance is remarkable, which is explained by the quick detection of incidents as the density of UAVs increases. However, the distance-based path planning algorithm remains stable since it establishes static paths (i.e., shortest paths) beforehand while neglecting unexpected incidents on the roads. Fig. 10(c) shows that the distance traveled in SEARCH is less than the time-based path planning algorithm. This is caused by the continuous collecting of information related to the roads by UAVs and 5G BSs, which can often find shorter distance paths when incidents suddenly happen.

To test the performance of SEARCH in terms of path

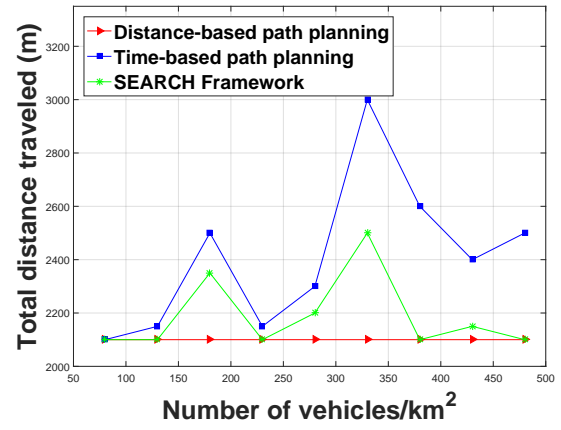

(a) Total distance $v s$. Density of vehicles (UAV density=16, Number of incidents=5).

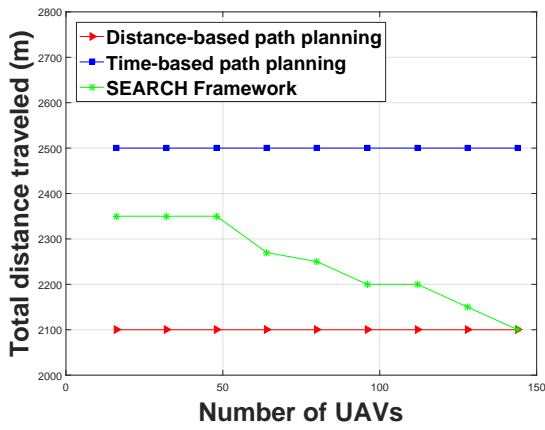

(b) Total distance vs. Density of UAVs (Vehicle density $=280$, Number of incidents $=5$ ).

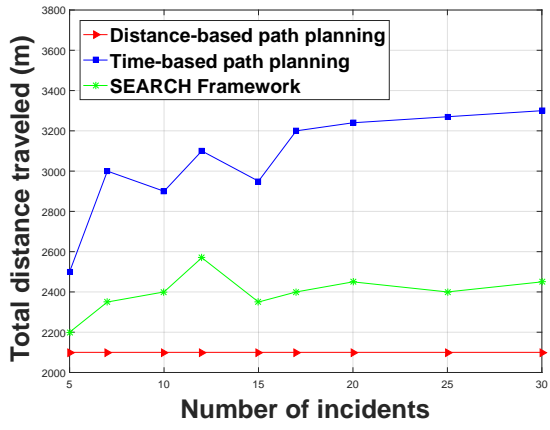

(c) Total distance $v s$. Number of incidents (Vehicle density=280, UAV density=16).

Fig. 10: Simulation results of traveled distance of SEARCH. 


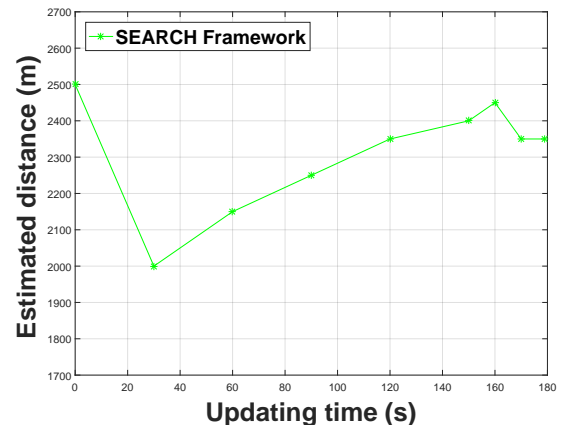

(a) Estimated distance vs. Updating time (Vehicle density $=280$, UAV density $=16$, Numbe of incidents $=5$ )

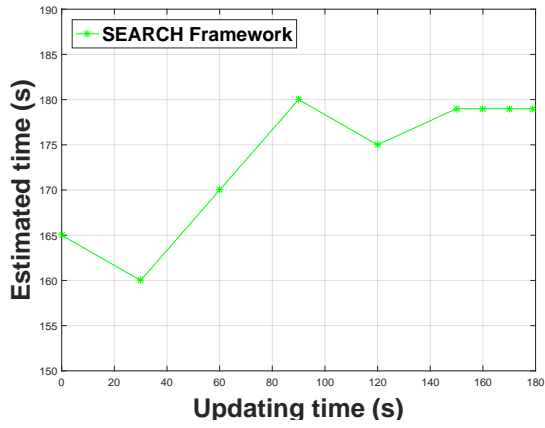

(b) Estimated time $v s$. Updating time

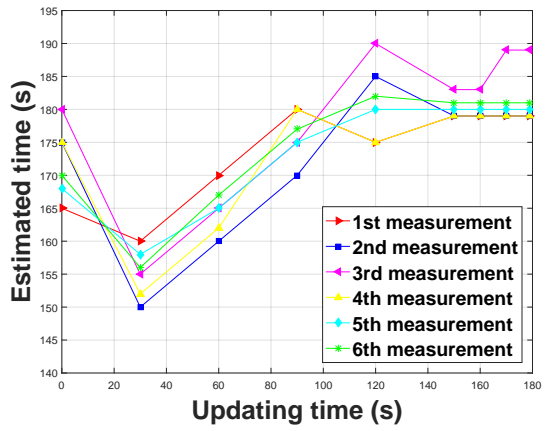

(c) Comparison of six time measurements (Vehicle density $=280$, UAV density $=16$, Number of incidents $=5$ ).

Fig. 11: Performance of path calculation dynamicity.

calculation dynamicity, we conduct different tests on the distance and vehicle travel time (c.f., Fig. 11). In fact, Fig. 11(a) shows the estimated distance to be traveled from the source to the destination at each crucial update. We distinguish that the distance is variable according to the traffic in the path. For example, the path is updated at times 30 and 60 seconds to be decreased until stabilization at time 170 and 180 seconds. As illustrated in Fig. 11(b), we calculate the time needed from the source to the destination at each update, impacting the already established path. We notice that the time to reach the destination at 0.00 seconds is estimated at 165 seconds. The time varies according to the path changes until it becomes stable. The stability is explained by not receiving any updates. Fig. 11(c)] shows a comparison between the estimated time of six path measurements. The estimated time of the first measurement is not considered as the most suitable path since other paths, including the shortest path in terms of distance, are obstructed at that time. When the vehicle makes the second update at the second intersection, it turns out that the second calculated path has the lowest estimated time. The vehicle continues receiving updates at each intersection until reaching the last intersection, in which the vehicle makes the sixth measurement to find out that the estimated time to destination is close to the second measurement (i.e., the lowest journey time).

The performance of the SEARCH framework architecture is analyzed and compared with two different architectures. The first architecture is a traditional approach in which every vehicle establishes communication directly with the BS without considering any SDN controllers. The second architecture is based on the method proposed in [18], named SD-VANET, in which every vehicle has to send signaling data to the closest vehicle to the BS. The performance of SEARCH architecture is investigated by analyzing the throughput, latency, control overhead, and processing latency. It should be stressed that UAVs and incidents are neglected during the evaluation of the mentioned above metrics.

As shown in Fig. 12(a), it is clear that SEARCH architecture significantly improves the throughput compared to other architectures. This improvement is explained by the provided high-data rates and direct communications of a large number of vehicles with UAVs while avoiding as much as possible 5G BSs. However, in SD-VANET, there are only specific vehicles that can establish a direct connection with BSs.

As in Fig. 12(b), it is clearly shown that the average latency achieved by SEARCH is significantly less than those produced by the traditional approach and SD-VANET. The reason is, in our proposed SEARCH architecture, the control functionality is divided among BSs and UAVs, thus reducing latency. Moreover, vehicles communicate directly with BSs and UAVs without V2V communications. Nevertheless, SD-VANET produces high latency compared to SEARCH with the increase of density of vehicles, which is explained by the complex handovers carried out between vehicles.

Fig. 12(c) shows that the control overhead has a proportional behavior according to the density of vehicles in all strategies. This is explained by the fact that control messages are periodically transmitted by vehicles to $5 \mathrm{G}$ BSs, which are automatically increased when the density of vehicles increases. Nevertheless, the different mechanisms applied by these approaches differentiate the control overhead levels between them. For instance, the traditional approach allows all vehicles to transmit their periodical messages to $5 \mathrm{G}$ BSs, which significantly deteriorates its performance. SD-VANET [18] has enhanced the performance of the traditional approach by selecting only the closest vehicles to make the transmission. As for SEARCH, vehicles tend to transmit as much as possible their periodical messages to existing UAVs in the sky, which significantly decreases the number of nodes that communicate with BSs, and thus reducing the overhead to their lowest level.

In Fig. 12(d), to accurately define the network utilization rate, we calculate the controller processing rate and the ratio of the total data arrival rate. It can be seen clearly that when the network load is low enough, processing latency tends to be close to zero for all the evaluated architectures. With the increase of the network utilization ratio, SEARCH still keeps the processing latency under $1 \mathrm{~ms}$ most of the time. Consequently, we can conclude that SEARCH meets the 5G latency requirement, which is not the case of SD-VANET and the traditional approach. 


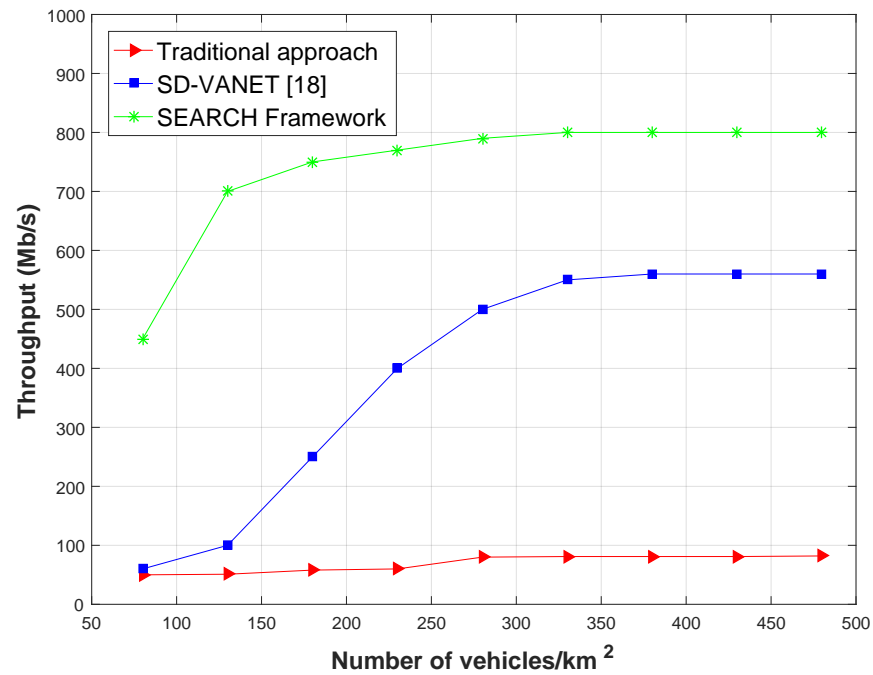

(a) Throughput vs. Density of vehicles.

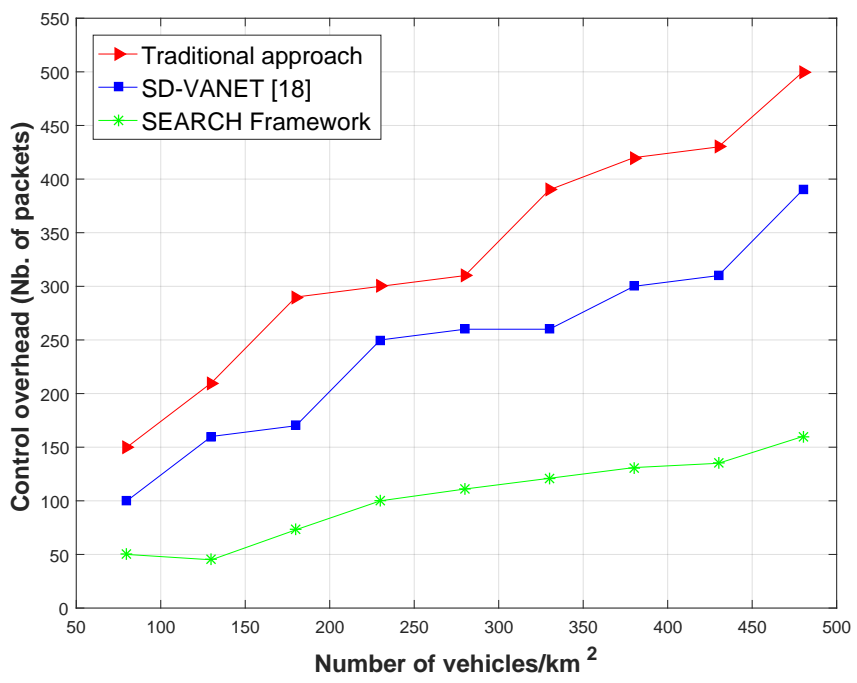

(c) Control overhead vs. Density of vehicles.

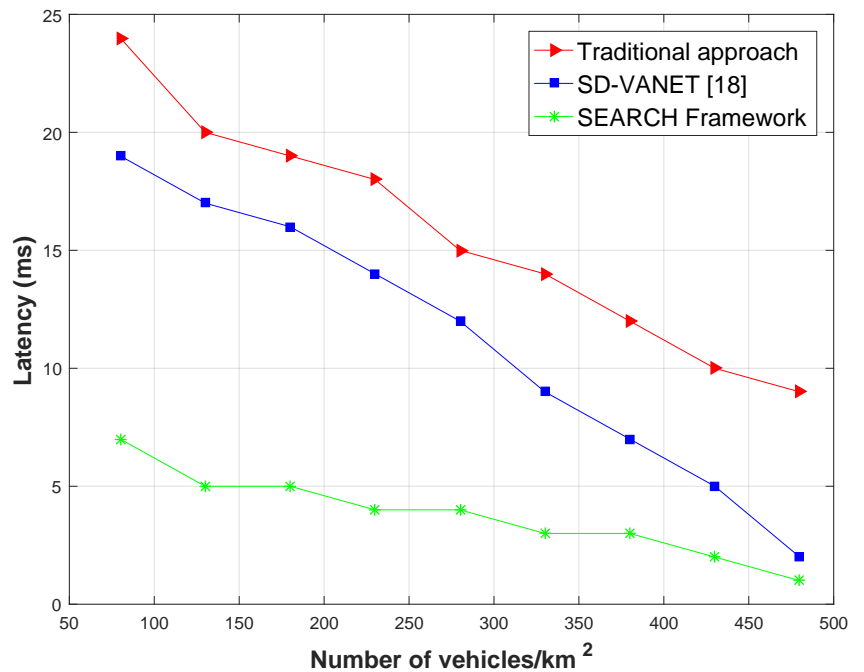

(b) Latency vs. Density of vehicles.

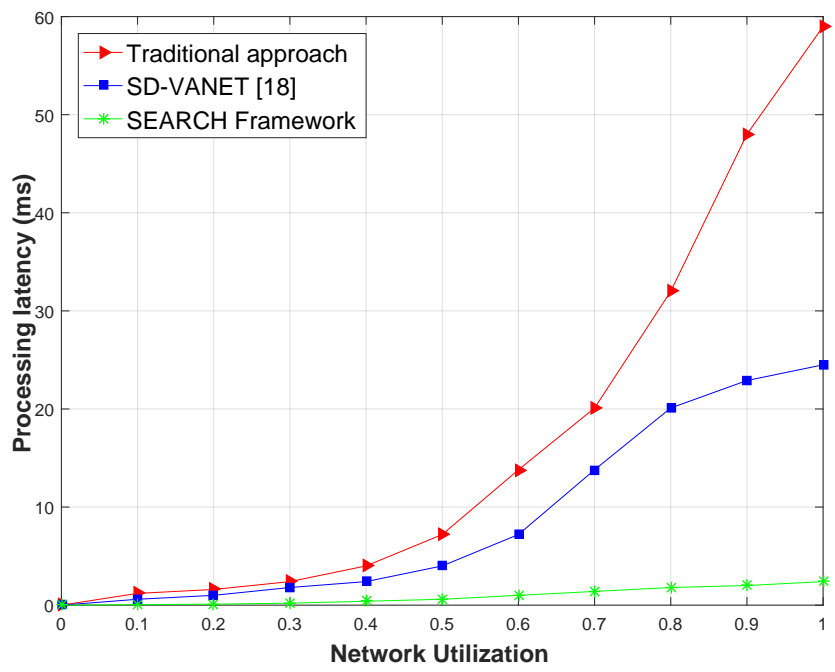

(d) Processing latency vs. Network utilization.

Fig. 12: Simulation results of our path planning algorithm.

\section{CONCLUSION}

This paper proposes a framework for dynamic path planning based on a low-latency SDN-based heterogeneous network called SEARCH. Due to the variable nature of congestion level and random incidents, SEARCH can dynamically adjust the best path of a vehicle during its journey. This solution can significantly improve the problem of static path planning by continuously collecting traffic information using $5 \mathrm{G} \mathrm{BSs}$ and UAVs deployed along roads. We exploit the Macroscopic model to calculate the journey time in each road segment and we use the Dijkstra algorithm to look for shorter time paths towards target destinations. Experimental analysis shows the robustness of the proposed framework in terms of decreasing journey times associated with the different road constraints in urban areas. However, we are aware of a certain number of limitations of our scheme, such as the deployment cost of aerial and terrestrial network equipment and the failure of our path planning algorithm when an accident occurs in front of a vehicle moving in a road segment. In the future, we will strongly seek to extend SEARCH with an enhanced network architecture by reducing the number of deployed infrastructures and replacing them with mobile entities, such as UAV-BSs. It will then be promising for us to enhance the path planning strategy based on predictive models and artificial intelligence (AI) techniques.

\section{ACKNOWLEDGMENT}

The authors would like to thank the Deanship of Scientific Research at Umm Al-Qura University for supporting this work by grant code 18-COM-1-01-0001.

\section{REFERENCES}

[1] L. C. Bento, R. Parafita, H. A. Rakha, and U. J. Nunes, "A study of the environmental impacts of intelligent automated vehicle control at intersections via V2V and V2I communications," Journal of Intelligent Transportation Systems, vol. 23, no. 1, pp. 41-59, 2019. 
[2] H. Teng, W. Liu, T. Wang, A. Liu, X. Liu, and S. Zhang, "A cost-efficient greedy code dissemination scheme through vehicle to sensing devices (V2SD) communication in smart city," IEEE Access, vol. 7, pp. 16675-16694, 2019.

[3] L. J. Basso, F. Feres, and H. E. Silva, "The efficiency of bus rapid transit (BRT) systems: A dynamic congestion approach," Transportation Research Part B: Methodological, vol. 127, pp. 47-71, 2019.

[4] I.-S. Comşa, S. Zhang, M. E. Aydin, P. Kuonen, Y. Lu, R. Trestian, and G. Ghinea, "Towards 5G: A reinforcement learning-based scheduling solution for data traffic management," IEEE Transactions on Network and Service Management, vol. 15, no. 4, pp. 1661-1675, 2018.

[5] T. Yamazato, N. Kawagita, H. Okada, T. Fujii, T. Yendo, S. Arai, and K. Kamakura, "The uplink visible light communication beacon system for universal traffic management," IEEE Access, vol. 5, pp. 22 282-22 290, 2017.

[6] I. Bisio, C. Garibotto, A. Grattarola, F. Lavagetto, and A. Sciarrone, "Smart and robust speaker recognition for context-aware in-vehicle applications," IEEE Transactions on Vehicular Technology, vol. 67, no. 9, pp. 8808-8821, 2018.

[7] M. Lourenço, F. S. Souza, C. M. Silva, R. I. Meneguette, and D. L. Guidoni, "A Hybrid V2I and V2V Approach for Urban Traffic Management in Vehicular Networks," in Proceedings of the Latin-American Conference on Communications (LATINCOM). IEEE, 2019 , pp. 1-6.

[8] O. S. Oubbati, N. Chaib, A. Lakas, P. Lorenz, and A. Rachedi, "UAV-assisted supporting services connectivity in urban VANETs," IEEE Transactions on Vehicular Technology, vol. 68, no. 4, pp. 3944-3951, 2019.

[9] O. S. Oubbati, M. Atiquzzaman, P. Lorenz, M. H. Tareque, and M. S. Hossain, "Routing in flying Ad Hoc networks: Survey, constraints, and future challenge perspectives," IEEE Access, vol. 7, pp. 81 057-81 105, 2019.

[10] O. S. Oubbati, M. Atiquzzaman, T. A. Ahanger, and A. Ibrahim, "Softwarization of UAV Networks: A Survey of Applications and Future Trends," IEEE Access, vol. 8, pp. $98073-98$ 125, 2020.

[11] A. Rego, L. Garcia, S. Sendra, and J. Lloret, "Software Defined Network-based control system for an efficient traffic management for emergency situations in smart cities," Future Generation Computer Systems, vol. 88, pp. 243-253, 2018.

[12] J. Li, D. Fu, Q. Yuan, H. Zhang, K. Chen, S. Yang, and F. Yang, "A traffic prediction enabled double rewarded value iteration network for route planning," IEEE Transactions on Vehicular Technology, vol. 68 , no. 5, pp. 4170-4181, 2019.

[13] X. Foukas, G. Patounas, A. Elmokashfi, and M. K. Marina, "Network slicing in 5G: Survey and challenges," IEEE Communications Magazine, vol. 55, no. 5, pp. 94-100, 2017.

[14] J. Bhatia, Y. Modi, S. Tanwar, and M. Bhavsar, "Software defined vehicular networks: A comprehensive review," International Journal of Communication Systems, vol. 32, no. 12, p. e4005, 2019.

[15] R. Hussain, F. Hussain, and S. Zeadally, "Integration of VANET and 5G Security: A review of design and implementation issues," Future Generation Computer Systems, vol. 101, pp. 843-864, 2019.

[16] I. Yaqoob, I. Ahmad, E. Ahmed, A. Gani, M. Imran, and N. Guizani, "Overcoming the key challenges to establishing vehicular communication: Is SDN the answer?" IEEE Communications Magazine, vol. 55, no. 7, pp. 128-134, 2017.

[17] F. Tang, Y. Kawamoto, N. Kato, and J. Liu, "Future intelligent and secure vehicular network toward 6G: Machine-learning approaches," Proceedings of the IEEE, vol. 108, no. 2, pp. 292-307, 2019.

[18] X. Ge, Z. Li, and S. Li, "5G software defined vehicular networks," IEEE Communications Magazine, vol. 55, no. 7, pp. 87-93, 2017.

[19] J. Liu, J. Wan, D. Jia, B. Zeng, D. Li, C.-H. Hsu, and H. Chen, "High-efficiency urban-traffic management in context-aware computing and 5G communication," IEEE Communications Magazine, vol. 55, no. 1, pp. 34-40, 2017.

[20] F. Tang, Z. M. Fadlullah, B. Mao, and N. Kato, "An intelligent traffic load prediction-based adaptive channel assignment algorithm in SDN-IoT: A deep learning approach," IEEE Internet of Things Journal, vol. 5, no. 6, pp. 5141-5154, 2018.

[21] S. Din, A. Paul, and A. Rehman, "5G-enabled Hierarchical architecture for software-defined intelligent transportation system," Computer Networks, vol. 150, pp. 81-89, 2019.

[22] S. Garg, K. Kaur, G. Kaddoum, S. H. Ahmed, and D. N. K. Jayakody, "SDN-based secure and privacy-preserving scheme for vehicular networks: A 5G perspective," IEEE Transactions on Vehicular Technology, vol. 68, no. 9, pp. 8421-8434, 2019.
[23] Z. He, J. Cao, and X. Liu, "SDVN: Enabling rapid network innovation for heterogeneous vehicular communication," IEEE network, vol. 30, no. 4, pp. 10-15, 2016.

[24] G. Xiaoxue et al., "Optimal route planning of parking lot based on dijkstra algorithm," in roceedings of the International Conference on Robots \& Intelligent System (ICRIS). IEEE, 2017, pp. 221-224.

[25] C. Zhao, M. Dong, K. Ota, J. Li, and J. Wu, "Edge-MapReduce-based intelligent information-centric IoV: Cognitive route planning," IEEE Access, vol. 7, pp. 50 549-50 560, 2019.

[26] J.-d. Zhang, Y.-j. Feng, F.-f. Shi, G. Wang, B. Ma, R.-s. Li, and X.-y. Jia, "Vehicle routing in urban areas based on the oil consumption weight-Dijkstra algorithm," IET Intelligent Transport Systems, vol. 10, no. 7, pp. 495-502, 2016.

[27] Z. Li, I. V. Kolmanovsky, E. M. Atkins, J. Lu, D. P. Filev, and Y. Bai, "Road disturbance estimation and cloud-aided comfort-based route planning," IEEE transactions on cybernetics, vol. 47, no. 11, pp. 3879-3891, 2016

[28] L. Yu, H. Jiang, and L. Hua, "Anti-Congestion Route Planning Scheme Based on Dijkstra Algorithm for Automatic Valet Parking System," Applied Sciences, vol. 9, no. 23, p. 5016, 2019.

[29] I. Bisio, C. Garibotto, F. Lavagetto, A. Sciarrone, and S. Zappatore, "Blind detection: Advanced techniques for WiFi-based drone surveillance," IEEE Transactions on Vehicular Technology, vol. 68, no. 1 , pp. 938-946, 2018

[30] W. Shi, H. Zhou, J. Li, W. Xu, N. Zhang, and X. Shen, "Drone assisted vehicular networks: Architecture, challenges and opportunities," IEEE Network, vol. 32, no. 3, pp. 130-137, 2018.

[31] G. K. Xilouris, M. C. Batistatos, G. E. Athanasiadou, G. Tsoulos, H. B. Pervaiz, and C. C. Zarakovitis, "UAV-assisted 5G network architecture with slicing and virtualization," in Proceedings of the IEEE Globecom Workshops (GC Wkshps). IEEE, 2018, pp. 1-7.

[32] V. Sharma, K. Srinivasan, H.-C. Chao, K.-L. Hua, and W.-H. Cheng, "Intelligent deployment of UAVs in 5G heterogeneous communication environment for improved coverage," Journal of Network and Computer Applications, vol. 85, pp. 94-105, 2017.

[33] M. Gapeyenko, V. Petrov, D. Moltchanov, S. Andreev, N. Himayat, and Y. Koucheryavy, "Flexible and reliable UAV-assisted backhaul operation in 5G mmWave cellular networks," IEEE Journal on Selected Areas in Communications, vol. 36, no. 11, pp. 2486-2496, 2018.

[34] S. Chen, J. Hu, Y. Shi, Y. Peng, J. Fang, R. Zhao, and L. Zhao, "Vehicle-to-everything (V2X) services supported by LTE-based systems and 5G," IEEE Communications Standards Magazine, vol. 1, no. 2, pp. 70-76, 2017.

[35] O. S. Oubbati, A. Lakas, P. Lorenz, M. Atiquzzaman, and A. Jamalipour, "Leveraging Communicating UAVs for Emergency Vehicle Guidance in Urban Areas," IEEE Transactions on Emerging Topics in Computing, 2019.

[36] R. Zhang, F. Yan, L. Shen, and Y. Wu, "A vehicle positioning method based on joint TOA and DOA estimation with V2R communications," in Proceedings of the IEEE 85th Vehicular Technology Conference (VTC Spring). IEEE, 2017, pp. 1-5.

[37] M. Van den Berg, A. Hegyi, B. De Schutter, and J. Hellendoorn, "A macroscopic traffic flow model for integrated control of freeway and urban traffic networks," in Proceedings of the 42nd IEEE International Conference on Decision and Control (IEEE Cat. No. 03CH37475), vol. 3. IEEE, 2003, pp. 2774-2779.

[38] A. Varga and R. Hornig, "An overview of the OMNeT++ simulation environment," in Proceedings of the 1st international conference on Simulation tools and techniques for communications, networks and systems \& workshops. ICST (Institute for Computer Sciences, Social-Informatics and ..., 2008, p. 60.

[39] M. Behrisch, L. Bieker, J. Erdmann, and D. Krajzewicz, "SUMO-simulation of urban mobility: an overview," in Proceedings of the Third International Conference on Advances in System Simulation (SIMUL 2011), 2011.

[40] S. M. Mousavi, H. R. Rabiee, M. Moshref, and A. Dabirmoghaddam, "Mobisim: A framework for simulation of mobility models in mobile ad-hoc networks," in Proceedings of the third IEEE International Conference on Wireless and Mobile Computing, Networking and Communications (WiMOB). IEEE, 2007, pp. 82-82.

[41] M. Haklay and P. Weber, "Openstreetmap: User-generated street maps," Ieee Pervas Comput, vol. 7, no. 4, pp. 12-18, 2008.

[42] C. Sommer, R. German, and F. Dressler, "Bidirectionally coupled network and road traffic simulation for improved IVC analysis," IEEE Transactions on mobile computing, vol. 10, no. 1, pp. 3-15, 2010. 


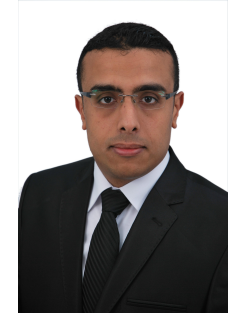

Omar Sami Oubbati is an Associate Professor at the Electronics department, University of Laghouat, Algeria and a Research Assistant in the Computer Science and Mathematics Lab (LIM) at the same university. He received his degree of Engineer (2010), M.Sc. in Computer Engineering (2011), M.Sc. degree (2014), and a $\mathrm{PhD}$ in Computer Science (2018). From Oct. 2016 to Oct. 2017, he was a Visiting Student with the Laboratory of Computer Science, University of Avignon, France. His main research interests are in Flying and Vehicular ad hoc networks, Energy harvesting and Mobile Edge Computing, Energy efficiency and Internet of Things (IoT). He is the recipient of the 2019 Best Survey Paper for Vehicular Communications (Elsevier). He has actively served as a reviewer for flagship IEEE Transactions journals and conferences, and participated as a Technical Program Committee Member for a variety of international conferences, such as IEEE ICC, IEEE CCNC, IEEE ICCCN, IEEE WCNC, IEEE ICAEE, and IEEE ICAIT. He serves on the editorial board of Vehicular Communications Journal of Elsevier and Communications Networks Journal of Frontiersin. He has also served as guest editor for a number of international journals. He is a member of the IEEE and IEEE Communications Society.

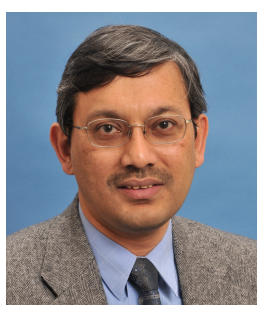

Mohammed Atiquzzaman received the M.S. and $\mathrm{Ph} . \mathrm{D}$. degrees in electrical engineering and electronics from the University of Manchester, U.K., in 1984 and 1987, respectively. He currently holds the Edith J. Kinney Gaylord Presidential Professorship with the School of Computer Science, University of Oklahoma, USA. His research has been funded by the National Science Foundation, National Aeronautics and Space Administration, U.S. Air Force, Cisco, and Honeywell. He co-authored Performance of TCP/IP Over ATM Networks and has authored over 300 refereed publications. His current research interests are in areas of transport protocols, wireless and mobile networks, ad hoc networks, satellite networks, power-aware networking, and optical communications. He CoChaired the IEEE High Performance Switching and Routing Symposium (2003, 2011), IEEE GLOBECOM and ICC (2014, 2012, 2010, 2009, 2007, and 2006), IEEE VTC (2013), and SPIE Quality of Service Over Next Generation Data Networks conferences (2001, 2002, and 2003). He was the Panels Co-Chair of INFOCOM'05, and has been on the program committee of many conferences, such as INFOCOM, GLOBECOM, ICCCN, ICCIT, Local Computer Networks, and serves on the review panels at the National Science Foundation. He was the Chair of the IEEE Communication Society Technical Committee on Communications Switching and Routing. He received the IEEE Communication Society's Fred W. Ellersick Prize and the NASA Group Achievement Award for outstanding work to further NASA Glenn Research Center's efforts in the area of the Advanced Communications/Air Traffic Management's Fiber Optic Signal Distribution for Aeronautical Communications project. He received from IEEE the 2018 Satellite and Space Communications Technical Recognition Award for valuable contributions to the Satellite and Space Communications scientific community. He also received the 2017 Distinguished Technical Achievement Award from IEEE Communications Society in recognition of outstanding technical contributions and services in the area of communications switching and routing. He is the Editor in Chief of Journal of Networks and Computer Applications, the founding Editor in Chief of Vehicular Communications, and serves served on the editorial boards of many journals, including IEEE Communications Magazine, IEEE Journal on Selected Areas in Communications, IEEE Transactions on Mobile Computing, Real Time Imaging Journal, Journal of Sensor Networks, and International Journal of Communication Systems.

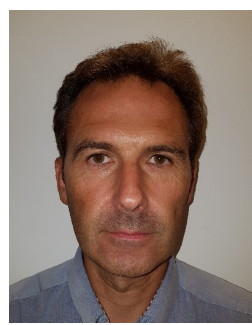

Pascal Lorenz received his M.Sc. (1990) and Ph.D. (1994) from the University of Nancy, France. Between 1990 and 1995 he was a research engineer at WorldFIP Europe and at Alcatel-Alsthom. He is a professor at the University of Haute-Alsace, France, since 1995. His research interests include QoS, wireless networks and high-speed networks. $\mathrm{He}$ is the author/co-author of 3 books, 3 patents and 200 international publications in refereed journals and conferences. He was Technical Editor of the IEEE Communications Magazine Editorial Board (2000-2006), IEEE Networks Magazine since 2015, IEEE Transactions on Vehicular Technology since 2017, Chair of IEEE ComSoc France (2014-2018), Financial chair of IEEE France (2017-2019), Chair of Vertical Issues in Communication Systems Technical Committee Cluster (2008-2009), Chair of the Communications Systems Integration and Modeling Technical Committee (2003-2009), Chair of the Communications Software Technical Committee (2008-2010) and Chair of the Technical Committee on Information Infrastructure and Networking (2016-2017). He has served as Co-Program Chair of IEEE WCNC'2012 and ICC'2004, Executive Vice-Chair of ICC'2017, Panel sessions co-chair for Globecom'16, tutorial chair of VTC'2013 Spring and WCNC'2010, track chair of PIMRC'2012 and WCNC'2014, symposium Co-Chair at Globecom 2007-2011, ICC 2008-2010, ICC'2014 and '2016. He is associate Editor for International Journal of Communication Systems (IJCS-Wiley), Journal on Security and Communication Networks (SCN-Wiley) and International Journal of Business Data Communications and Networking, Journal of Network and Computer Applications (JNCA-Elsevier). He is senior member of the IEEE, IARIA fellow and member of many international program committees. He was IEEE ComSoc Distinguished Lecturer Tour during 2013-2014.

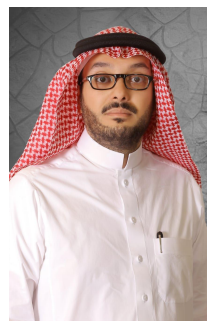

Abdullah Baz received the B.Sc. degree in electrical and computer engineering from UQU, in 2002, the M.Sc. degree in electrical and computer engineering from KAU, in 2007, and the M.Sc. degree in communication and signal processing and the Ph.D. degree in computer system design from Newcastle University, in 2009 and 2014, respectively. He was a Vice-Dean, and then the Dean of the Deanship of Scientific Research with UQU, from 2014 to 2020. He is currently an Associate Professor with the Computer Engineering Department, a Vice-Dean of DFMEA, the General Director of the Decision Support Center, and the Consultant of the University Vice Chancellor with UQU. His research interests include data science, ML, AI, VLSI design, EDA/CAD tools, intelligent transportation, computer system and architecture, smart systems, smart health. Since 2015, he has been served as a Review Committee Member of the IEEE International Symposium on Circuits and Systems (ISCAS) and a member of the Technical Committee of the IEEE VLSI Systems and Applications. In 2017, IEEE has elevated him to the grade of IEEE Senior Member. $\mathrm{He}$ served as a Reviewer in a number of journals, including the IEEE Internet of Things, the IET Computer Vision, the Artificial Intelligence Review, IEEE Access, and the IET Circuits, Devices and Systems.

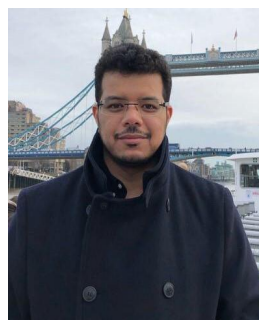

Hosam Alhakami received the B.Sc. degree in computer science from King Abdulaziz University, Saudi Arabia, in 2004, the M.Sc. degree in internet software systems from Birmingham University, Birmingham, U.K., in 2009, and the Ph.D. degree in software engineering from De Montfort University, in 2015. From 2004 to 2007, he worked with Software Development Industry, where he implemented several systems and solutions for a national academic institution. Dr. Alhakami was the Vice-Dean of the Deanship of Admission and Registration for Academic affairs with UQU, from 2015 to 2020. Currently, he is an associate professor of the computer science department with UQU. His research interests include algorithms, semantic web, and optimization techniques. He focuses on enhancing real-world matching systems using machine learning and data analytics in a context of supporting decision-making. 